\title{
NEW ERROR INEQUALITIES FOR THE LAGRANGE INTERPOLATING POLYNOMIAL
}

NENAD UJEVIĆ

Received 30 August 2005

A new representation of remainder of Lagrange interpolating polynomial is derived. Error inequalities of Ostrowski-Grüss type for the Lagrange interpolating polynomial are established. Some similar inequalities are also obtained.

\section{Introduction}

Many error inequalities in polynomial interpolation can be found in $[1,7]$. These error bounds for interpolating polynomials are usually expressed by means of the norms $\|\cdot\|_{p}, 1 \leq p \leq \infty$. Some new error inequalities (for corrected interpolating polynomials) are given in $[10,11]$. The last mentioned inequalities are similar to error inequalities obtained in recent years in numerical integration and they are known in the literature as inequalities of Ostrowski (or Ostrowski-like, Ostrowski-Grüss) type. For example, in [9] we can find inequalities of Ostrowski-Grüss type for the well-known Simpson's quadrature rule,

$$
\left|\int_{x_{0}}^{x_{2}} f(t) d t-\frac{h}{3}\left[f\left(x_{0}\right)+4 f\left(x_{1}\right)+f\left(x_{2}\right)\right]\right| \leq C_{n}\left(\Gamma_{n}-\gamma_{n}\right) h^{n+1},
$$

where $x_{i}=x_{0}+i h$, for $h>0, i=1,2, \gamma_{n}, \Gamma_{n}$ are real numbers such that $\gamma_{n} \leq f^{(n)}(t) \leq \Gamma_{n}$, for all $t \in\left[x_{0}, x_{2}\right]$, and $C_{n}$ are constants, $n \in\{1,2,3\}$.

The inequalities of Ostrowski type can be also found in $[2,3,4,5,6,12]$. In some of the mentioned papers, we can find estimations for errors of quadrature formulas which are expressed by means of the differences $\Gamma_{k}-\gamma_{k}, S-\gamma_{k}, \Gamma_{k}-S$, where $\Gamma_{k}, \gamma_{k}$ are real numbers such that $\gamma_{k} \leq f^{(k)}(t) \leq \Gamma_{k}, t \in[a, b]$ ( $k$ is a positive integer while $[a, b]$ is an interval of integration) and $S=\left[f^{(k-1)}(b)-f^{(k-1)}(a)\right] /(b-a)$. It is shown that the estimations expressed in such a way can be much better than the estimations expressed by means of the norms $\left\|f^{(k)}\right\|_{p}, 1 \leq p \leq \infty$.

As we know there is a close relationship between interpolation polynomials and quadrature rules. Thus, it is a natural try to establish similar error inequalities in polynomial interpolation. 
We first establish general error inequalities, expressed by means of $\left\|f^{(k)}-P_{m}\right\|$, where $P_{m}$ is any polynomial of degree $m$ and then we obtain inequalities of the above mentioned types. For that purpose, we derive a new representation of remainder of the interpolating polynomial. This is done in Section 2. In Section 3, we obtain the error inequalities of the above-mentioned types. In Section 4, we give some results for derivatives.

Finally, we emphasize that the usual error inequalities in polynomial interpolation (for the Lagrange interpolating polynomial $\left.L_{n}(x)\right)$ are given by means of the $(n+1)$ th derivative while in this paper we can find these error inequalities expressed by means of the $k$ th derivative for $k=1,2, \ldots, n$.

\section{Representation of remainder}

Let $D=\left\{a=x_{0}<x_{1}<\cdots<x_{n}=b\right\}$ be a given subdivision of the interval $[a, b]$ and let $f:[a, b] \rightarrow \mathbb{R}$ be a given function. The Lagrange interpolation polynomial is given by

$$
L_{n}(x)=\sum_{i=0}^{n} p_{n i}(x) f\left(x_{i}\right)
$$

where

$$
p_{n i}(x)=\frac{\left(x-x_{0}\right) \cdots\left(x-x_{i-1}\right)\left(x-x_{i+1}\right) \cdots\left(x-x_{n}\right)}{\left(x_{i}-x_{0}\right) \cdots\left(x_{i}-x_{i-1}\right)\left(x_{i}-x_{i+1}\right) \cdots\left(x_{i}-x_{n}\right)}
$$

for $i=0,1, \ldots, n$. We have the Cauchy relations [7, pages 160-161],

$$
\begin{gathered}
\sum_{i=0}^{n} p_{n i}(x)=1, \\
\sum_{i=0}^{n} p_{n i}(x)\left(x-x_{i}\right)^{j}=0, \quad j=1,2, \ldots, n .
\end{gathered}
$$

Let $\bar{D}=\left\{x_{0}=a<x_{1}<\cdots<x_{n}=b\right\}$ be a given uniform subdivision of the interval $[a, b]$, that is, $x_{i}=x_{0}+i h, h=(b-a) / n, i=0,1,2, \ldots, n$. Then the Lagrange interpolating polynomial is given by

$$
L_{n}(x)=L_{n}\left(x_{0}+t h\right)=(-1)^{n} \frac{t(t-1) \cdots(t-n)}{n !} \sum_{i=0}^{n}(-1)^{i}\left(\begin{array}{l}
n \\
i
\end{array}\right) \frac{f\left(x_{i}\right)}{t-i}
$$

where $t \notin\{0,1,2, \ldots, n\}, 0<t<n$.

Lemma 2.1. Let $P_{m}(t)$ be an arbitrary polynomial of degree $\leq m$ and let $p_{n i}(x)$ be defined by (2.2). Then

$$
\sum_{i=0}^{n} p_{n i}(x) \int_{x_{i}}^{x} P_{m}(t)\left(t-x_{i}\right)^{k} d t=0
$$

for $0 \leq k+m \leq n-1$ and $x \in[a, b]$. 
Proof. Let $x$ be a given real number. Then we have

$$
P_{m}(t)=\sum_{j=0}^{m} c_{j}(x-t)^{j}
$$

for some coefficients $c_{j}=c_{j}(x), j=0,1,2, \ldots, m$. (This is a consequence of the Taylor formula.) Thus,

$$
\sum_{i=0}^{n} p_{n i}(x) \int_{x_{i}}^{x} P_{m}(t)\left(t-x_{i}\right)^{k} d t=\sum_{j=0}^{m} c_{j} \sum_{i=0}^{n} p_{n i}(x) \int_{x_{i}}^{x}(x-t)^{j}\left(t-x_{i}\right)^{k} d t .
$$

Let $\beta(\cdot, \cdot)$ and $\Gamma(\cdot)$ denote the beta and gamma functions, respectively. We now calculate

$$
\begin{aligned}
\int_{x_{i}}^{x}(x-t)^{j}\left(t-x_{i}\right)^{k} d t & =\int_{0}^{x-x_{i}}\left(x-x_{i}-u\right)^{j} u^{k} d u \\
& =\left(x-x_{i}\right)^{j} \int_{0}^{x-x_{i}}\left(1-\frac{u}{x-x_{i}}\right)^{j} u^{k} d u \\
& =\left(x-x_{i}\right)^{j+k+1} \int_{0}^{1}(1-v)^{j} v^{k} d v \\
& =\beta(j+1, k+1)\left(x-x_{i}\right)^{j+k+1} \\
& =\frac{\Gamma(k+1) \Gamma(j+1)}{\Gamma(k+j+2)}\left(x-x_{i}\right)^{j+k+1} \\
& =\frac{k ! j !}{(k+j+1) !}\left(x-x_{i}\right)^{j+k+1} .
\end{aligned}
$$

From (2.8) and (2.9) it follows that

$$
\sum_{i=0}^{n} p_{n i}(x) \int_{x_{i}}^{x} P_{m}(t)\left(t-x_{i}\right)^{k} d t=\sum_{j=0}^{m} c_{j} \frac{k ! j !}{(k+j+1) !} \sum_{i=0}^{n} p_{n i}(x)\left(x-x_{i}\right)^{j+k+1} .
$$

From (2.10) and (2.4) we conclude that (2.6) holds.

Theorem 2.2. Let $f \in C^{n+1}(a, b)$ and let the assumptions of Lemma 2.1 hold. Then

$$
f(x)=L_{n}(x)+R_{k, m}(x),
$$

where $L_{n}(x)$ is given by (2.1) and

$$
R_{k, m}(x)=\frac{(-1)^{k}}{k !} \sum_{i=0}^{n} p_{n i}(x) \int_{x_{i}}^{x}\left[f^{(k+1)}(t)-P_{m}(t)\right]\left(t-x_{i}\right)^{k} d t
$$

Proof. We have

$$
R_{k, m}(x)=\frac{(-1)^{k}}{k !} \sum_{i=0}^{n} p_{n i}(x) \int_{x_{i}}^{x} f^{(k+1)}(t)\left(t-x_{i}\right)^{k} d t-\frac{(-1)^{k}}{k !} \sum_{i=0}^{n} p_{n i}(x) \int_{x_{i}}^{x} P_{m}(t)\left(t-x_{i}\right)^{k} d t .
$$


3838 Inequalities in polynomial interpolation

From (2.13) and (2.6) it follows that

$$
R_{k, m}(x)=R_{k}(x)=\frac{(-1)^{k}}{k !} \sum_{i=0}^{n} p_{n i}(x) \int_{x_{i}}^{x} f^{(k+1)}(t)\left(t-x_{i}\right)^{k} d t .
$$

For $k=0$ we have

$$
\begin{aligned}
R_{0}(x) & =\sum_{i=0}^{n} p_{n i}(x) \int_{x_{i}}^{x} f^{\prime}(t) d t \\
& =\sum_{i=0}^{n} p_{n i}(x)\left[f(x)-f\left(x_{i}\right)\right]=f(x)-L_{n}(x),
\end{aligned}
$$

since (2.3) holds.

We now suppose that $k \geq 1$. Integrating by parts, we obtain

$$
\frac{(-1)^{k}}{k !} \int_{x_{i}}^{x} f^{(k+1)}(t)\left(t-x_{i}\right)^{k} d t=\frac{(-1)^{k}}{k !} f^{(k)}(x)\left(x-x_{i}\right)^{k}+\frac{(-1)^{k-1}}{(k-1) !} \int_{x_{i}}^{x} f^{(k)}(t)\left(t-x_{i}\right)^{k-1} d t
$$

In a similar way we get

$$
\begin{aligned}
& \frac{(-1)^{k-1}}{(k-1) !} \int_{x_{i}}^{x} f^{(k)}(t)\left(t-x_{i}\right)^{k-1} d t \\
& \quad=\frac{(-1)^{k-1}}{(k-1) !} f^{(k-1)}(x)\left(x-x_{i}\right)^{k-1} \frac{(-1)^{k-2}}{(k-2) !} \int_{x_{i}}^{x} f^{(k-1)}(t)\left(t-x_{i}\right)^{k-2} d t .
\end{aligned}
$$

Continuing in this way, we get

$$
\begin{aligned}
\frac{(-1)^{k}}{k !} \int_{x_{i}}^{x} f^{(k+1)}(t)\left(t-x_{i}\right)^{k} d t & =\sum_{j=1}^{k} \frac{(-1)^{j}}{j !} f^{(j)}(x)\left(x-x_{i}\right)^{j}+\int_{x_{i}}^{x} f^{\prime}(t) d t \\
& =f(x)-f\left(x_{i}\right)+\sum_{j=1}^{k} \frac{(-1)^{j}}{j !} f^{(j)}(x)\left(x-x_{i}\right)^{j} .
\end{aligned}
$$

From (2.14) and (2.18) it follows that

$$
\begin{aligned}
R_{k}(x) & =\sum_{i=0}^{n} p_{n i}(x)\left[f(x)-f\left(x_{i}\right)+\sum_{j=1}^{k} \frac{(-1)^{j}}{j !} f^{(j)}(x)\left(x-x_{i}\right)^{j}\right] \\
& =f(x)-L_{n}(x)+\sum_{j=1}^{k} \frac{(-1)^{j}}{j !} f^{(j)}(x) \sum_{i=0}^{n} p_{n i}(x)\left(x-x_{i}\right)^{j} \\
& =f(x)-L_{n}(x), \quad k=1,2, \ldots, n,
\end{aligned}
$$

since (2.3) and (2.4) hold. From (2.14), (2.15), and (2.19) we see that (2.11) holds. 


\section{Error inequalities}

We now introduce the notations

$$
\begin{aligned}
\omega_{n}(x) & =\left(x-x_{0}\right)\left(x-x_{1}\right) \cdots\left(x-x_{n}\right), \\
C_{k}(x) & =\sum_{i=0}^{n} \frac{\left|x-x_{i}\right|^{k}}{\left|x_{i}-x_{0}\right| \cdots\left|x_{i}-x_{i-1}\right|\left|x_{i}-x_{i+1}\right| \cdots\left|x_{i}-x_{n}\right|}, \\
B_{k}(x) & =\sum_{i=0}^{n} \frac{\left(S_{k i}-y_{k+1}\right)\left|x-x_{i}\right|^{k}}{\left|x_{i}-x_{0}\right| \cdots\left|x_{i}-x_{i-1}\right|\left|x_{i}-x_{i+1}\right| \cdots\left|x_{i}-x_{n}\right|}, \\
D_{k}(x) & =\sum_{i=0}^{n} \frac{\left(\Gamma_{k+1}-S_{k i}\right)\left|x-x_{i}\right|^{k}}{\left|x_{i}-x_{0}\right| \cdots\left|x_{i}-x_{i-1}\right|\left|x_{i}-x_{i+1}\right| \cdots\left|x_{i}-x_{n}\right|},
\end{aligned}
$$

where $S_{k i}=\left[f^{(k)}(x)-f^{(k)}\left(x_{i}\right)\right] /\left(x-x_{i}\right), i=0,1, \ldots, n$, and $\gamma_{k+1}, \Gamma_{k+1}$ are real numbers such that $\gamma_{k+1} \leq f^{(k+1)}(t) \leq \Gamma_{k+1}, t \in[a, b], k=0,1, \ldots, n-1$.

Let $g \in C(a, b)$. As we know among all algebraic polynomials of degree $\leq m$ there exists the only polynomial $P_{m}^{*}(t)$ having the property that

$$
\left\|g-P_{m}^{*}\right\|_{\infty} \leq\left\|g-P_{m}\right\|_{\infty}
$$

where $P_{m} \in \Pi_{m}$ is an arbitrary polynomial of degree $\leq m$. We define

$$
E_{m}(g)=\left\|g-P_{m}^{*}\right\|=\inf _{P_{m} \in \Pi_{m}}\left\|g-P_{m}\right\|_{\infty} .
$$

THeOREm 3.1. Under the assumptions of Theorem 2.2,

$$
\left|f(x)-L_{n}(x)\right| \leq \frac{E_{m}\left(f^{(k+1)}\right)}{(k+1) !} C_{k}(x)\left|\omega_{n}(x)\right|,
$$

where $C_{k}(\cdot)$ and $E_{m}(\cdot)$ are defined by (3.2) and (3.6), respectively.

Proof. Let $P_{m}(t)=P_{m}^{*}(t)$, where $P_{m}^{*}(t)$ is defined by (3.6) for the function $g(t)=f^{(k+1)}(t)$. We have

$$
\begin{aligned}
\left|R_{k, m}(x)\right| & =\left|\frac{(-1)^{k}}{k !} \sum_{i=0}^{n} p_{n i}(x) \int_{x_{i}}^{x}\left[f^{(k+1)}(t)-P_{m}^{*}(t)\right]\left(t-x_{i}\right)^{k} d t\right| \\
& \leq \frac{\left\|f^{(k+1)}-P_{m}^{*}\right\|_{\infty}}{(k+1) !} C_{k}(x)\left|\omega_{n}(x)\right| \\
& =\frac{E_{m}\left(f^{(k+1)}\right)}{(k+1) !} C_{k}(x)\left|\omega_{n}(x)\right|,
\end{aligned}
$$

since

$$
\left|\int_{x_{i}}^{x}\left(t-x_{i}\right)^{k} d t\right|=\frac{\left|x-x_{i}\right|^{k+1}}{k+1} .
$$


3840 Inequalities in polynomial interpolation

Remark 3.2. The above estimate has only theoretical importance, since it is difficult to find the polynomial $P^{*}$. In fact, we can find $P^{*}$ only for some special cases of functions. However, we can use the estimate to obtain some practical estimations_-see Theorem 3.3.

THeOREM 3.3. Let the assumptions of Theorem 2.2 hold. If $\gamma_{k+1}, \Gamma_{k+1}$ are real numbers such that $\gamma_{k+1} \leq f^{(k+1)}(t) \leq \Gamma_{k+1}, t \in[a, b], k=0,1, \ldots, n-1$, then

$$
\left|f(x)-L_{n}(x)\right| \leq \frac{\Gamma_{k+1}-\gamma_{k+1}}{2(k+1) !} C_{k}(x)\left|\omega_{n}(x)\right|
$$

where $\omega_{n}$ and $C_{k}(\cdot)$ are defined by (3.1) and (3.2), respectively. Also

$$
\begin{aligned}
& \left|f(x)-L_{n}(x)\right| \leq \frac{\left|\omega_{n}(x)\right|}{k !} B_{k}(x), \\
& \left|f(x)-L_{n}(x)\right| \leq \frac{\left|\omega_{n}(x)\right|}{k !} D_{k}(x),
\end{aligned}
$$

where $B_{k}(\cdot)$ and $D_{k}(\cdot)$ are defined by (3.3) and (3.4), respectively.

Proof. We set $P_{m}(t)=\left(\Gamma_{k+1}+\gamma_{k+1}\right) / 2$ in (2.12). Then we have

$$
\left|f(x)-L_{n}(x)\right|=\left|R_{k}(x)\right| \leq \frac{1}{k !} \sum_{i=0}^{n}\left|p_{n i}(x)\right||| f^{(k+1)}-\frac{\Gamma_{k+1}+\gamma_{k+1}}{2} \|_{\infty}\left|\int_{x_{i}}^{x}\left(t-x_{i}\right)^{k} d t\right| .
$$

We also have

$$
\begin{aligned}
\left\|f^{(k+1)}-\frac{\Gamma_{k+1}+\gamma_{k+1}}{2}\right\|_{\infty} \leq \frac{\Gamma_{k+1}-\gamma_{k+1}}{2}, \\
\left|\int_{x_{i}}^{x}\left(t-x_{i}\right)^{k} d t\right|=\frac{\left|x-x_{i}\right|^{k+1}}{k+1} .
\end{aligned}
$$

From the above three relations we get

$$
\begin{aligned}
\left|f(x)-L_{n}(x)\right| & \leq \frac{\Gamma_{k+1}-\gamma_{k+1}}{2(k+1) !} \sum_{i=0}^{n}\left|p_{n i}(x)\right|\left|x-x_{i}\right|^{k+1} \\
& =\frac{\Gamma_{k+1}-\gamma_{k+1}}{2(k+1) !} C_{k}(x)\left|\omega_{n}(x)\right| .
\end{aligned}
$$

The first inequality is proved.

We now set $P_{m}(t)=\gamma_{k+1}$ in (2.12). Then we have

$$
\left|f(x)-L_{n}(x)\right|=\left|R_{k}(x)\right| \leq \frac{1}{k !} \sum_{i=0}^{n}\left|p_{n i}(x)\right|\left|\int_{x_{i}}^{x}\left[f^{(k+1)}(t)-\gamma_{k+1}\right]\left(t-x_{i}\right)^{k} d t\right| .
$$


We also have

$$
\begin{aligned}
\left|\int_{x_{i}}^{x}\left[f^{(k+1)}(t)-\gamma_{k+1}\right]\left(t-x_{i}\right)^{k} d t\right| & \leq\left|x-x_{i}\right|^{k}\left|f^{(k)}(x)-f^{(k)}\left(x_{i}\right)-\gamma_{k+1}\left(x-x_{i}\right)\right| \\
& =\left|x-x_{i}\right|^{k+1}\left(S_{k i}-\gamma_{k+1}\right) .
\end{aligned}
$$

Thus,

$$
\begin{aligned}
\left|f(x)-L_{n}(x)\right| & \leq \frac{1}{k !} \sum_{i=0}^{n}\left|p_{n i}(x)\right|\left|x-x_{i}\right|^{k+1}\left(S_{k i}-\gamma_{k+1}\right) \\
& =\frac{\left|\omega_{n}(x)\right|}{k !} B_{k}(x) .
\end{aligned}
$$

The second inequality is proved. In a similar way we prove that the third inequality holds.

Lemma 3.4. Let $D=\left\{x_{0}=a<x_{1}<\cdots<x_{n}=b\right\}$ be a given uniform subdivision of the interval $[a, b]$, that is, $x_{i}=x_{0}+i h, h=(b-a) / n, i=0,1,2, \ldots, n$. If $x \in\left(x_{j-1}, x_{j}\right)$, for some $j \in\{1,2, \ldots, n\}$, then

$$
\begin{gathered}
\left|\omega_{n}(x)\right| \leq j !(n-j+1) ! h^{n+1}, \\
C_{k}(x) \leq \frac{2^{n}}{n !}\left\{\frac{1}{2}[n+1+|n-2 j+1|]\right\}^{k} h^{k-n}, \\
C_{k}(x)\left|\omega_{n}(x)\right| \leq \alpha_{j n k} \frac{n-j+1}{n} \frac{2^{n}(b-a)^{k+1}}{\left(\begin{array}{l}
n \\
j
\end{array}\right)},
\end{gathered}
$$

where

$$
\alpha_{j n k}=\left[\frac{1}{2 n}(n+1+|2 j-n-1|)\right]^{k} .
$$

This lemma is proved in [10].

Remark 3.5. Note that

$$
\alpha_{j n k} \leq 1
$$

and $\alpha_{j n k}=1$ if and only if $j=1$ or $j=n$. If we choose $x \in\left[x_{j}, x_{j+1}\right], j=0,1, \ldots, n-1$, then we get the factor $(j+1) / n$ instead of the factor $(n-j+1) / n$ in (3.20).

Theorem 3.6. Under the assumptions of Lemma 3.4 and Theorem 3.3,

$$
\left|f(x)-L_{n}(x)\right| \leq \frac{\Gamma_{k+1}-\gamma_{k+1}}{(k+1) !} \alpha_{j n k} \frac{n-j+1}{n} \frac{2^{n-1}(b-a)^{k+1}}{\left(\begin{array}{l}
n \\
j
\end{array}\right)} .
$$

Proof. The proof follows immediately from Theorem 3.3 and Lemma 3.4. 
3842 Inequalities in polynomial interpolation

\section{Results for derivatives}

LEMma 4.1. Let $1 \leq j \leq n-1$ and $j+1 \leq r \leq n$. Then

$$
\sum_{i=0}^{n} p_{n i}^{(j)}(x)\left(x-x_{i}\right)^{r}=0 .
$$

Proof. We have (see (2.4))

$$
A(x)=\sum_{i=0}^{n} p_{n i}(x)\left(x-x_{i}\right)^{r}=0, \quad \text { for } 1 \leq r \leq n .
$$

Thus,

$$
A^{\prime}(x)=\sum_{i=0}^{n} p_{n i}^{\prime}(x)\left(x-x_{i}\right)^{r}+r \sum_{i=0}^{n} p_{n i}(x)\left(x-x_{i}\right)^{r-1}=0,
$$

if $1 \leq r \leq n$. If $n \geq r-1 \geq 1$, that is, $n+1 \geq r \geq 2$, then

$$
r \sum_{i=0}^{n} p_{n i}(x)\left(x-x_{i}\right)^{r-1}=0 .
$$

From (4.3) and (4.4) we get

$$
\sum_{i=0}^{n} p_{n i}^{\prime}(x)\left(x-x_{i}\right)^{r}=0, \quad \text { for } 2 \leq r \leq n .
$$

(Note that $\{r: 1 \leq r \leq n\} \cap\{r: 2 \leq r \leq n+1\}=\{r: 2 \leq r \leq n\}$. Here we use this fact and similar facts without a special mentioning.)

We now suppose that

$$
\sum_{i=0}^{n} p_{n i}^{(j)}(x)\left(x-x_{i}\right)^{r}=0,
$$

for $j=1,2, \ldots, m, m<n-1$ and $j+1 \leq r \leq n$. We wish to prove that

$$
\sum_{i=0}^{n} p_{n i}^{(m+1)}(x)\left(x-x_{i}\right)^{r}=0, \quad \text { for } m+2 \leq r \leq n .
$$

For that purpose, we first calculate

$$
\begin{aligned}
A^{(m)}(x) & =\sum_{i=0}^{n}\left[p_{n i}(x)\left(x-x_{i}\right)^{r}\right]^{(m)} \\
& =\sum_{i=0}^{n} \sum_{k=0}^{m}\left(\begin{array}{l}
m \\
k
\end{array}\right) p_{n i}^{(k)}(x) \frac{r !}{(r-m+k) !}\left(x-x_{i}\right)^{r-m+k} \\
& =\sum_{k=0}^{m}\left(\begin{array}{c}
m \\
k
\end{array}\right) \frac{r !}{(r-m+k) !} \sum_{i=0}^{n} p_{n i}^{(k)}(x)\left(x-x_{i}\right)^{r-m+k}
\end{aligned}
$$


We have

$$
A^{(m)}(x)=0, \quad \text { for } r \geq m+1,
$$

by the above assumption. Thus,

$$
A^{(m+1)}(x)=0 .
$$

On the other hand, we have

$$
\begin{aligned}
A^{(m+1)}(x)= & \frac{d}{d x} A^{(m)}(x) \\
= & \sum_{k=0}^{m}\left(\begin{array}{c}
m \\
k
\end{array}\right) \frac{r !}{(r-m+k) !} \sum_{i=0}^{n} p_{n i}^{(k+1)}(x)\left(x-x_{i}\right)^{r-m+k} \\
& +\sum_{k=0}^{m}\left(\begin{array}{c}
m \\
k
\end{array}\right) \frac{r !}{(r-m+k-1) !} \sum_{i=0}^{n} p_{n i}^{(k)}(x)\left(x-x_{i}\right)^{r-m+k-1} \\
= & 0 .
\end{aligned}
$$

We now rewrite the above relation in the form

$$
\begin{gathered}
\sum_{i=0}^{n} p_{n i}^{(m+1)}(x)\left(x-x_{i}\right)^{r}+\sum_{k=0}^{m-1}\left(\begin{array}{c}
m \\
k
\end{array}\right) \frac{r !}{(r-m+k) !} \sum_{i=0}^{n} p_{n i}^{(k+1)}(x)\left(x-x_{i}\right)^{r-m+k} \\
+\sum_{k=0}^{m}\left(\begin{array}{c}
m \\
k
\end{array}\right) \frac{r !}{(r-m+k-1) !} \sum_{i=0}^{n} p_{n i}^{(k)}(x)\left(x-x_{i}\right)^{r-m+k-1}=0 .
\end{gathered}
$$

For $r-m+k-1 \geq k+1$, that is, $r \geq m+2$, we have

$$
\sum_{i=0}^{n} p_{n i}^{(k)}(x)\left(x-x_{i}\right)^{r-m+k-1}=0
$$

by the above assumption. We also have

$$
\sum_{i=0}^{n} p_{n i}^{(k+1)}(x)\left(x-x_{i}\right)^{r-m+k}=0
$$

if $r-m+k \geq k+2$, that is, $r \geq m+2$. Thus (4.7) holds. This completes the proof.

Theorem 4.2. Let $f \in C^{n+1}(a, b)$ and let $P_{r}(t)$ be an arbitrary polynomial of degree $\leq r$ and let $0 \leq k \leq n, 1 \leq m \leq k$. Then

$$
f^{(m)}(x)=L_{n}^{(m)}(x)+E_{k, r}(x)
$$

where

$$
E_{k, r}(x)=\frac{(-1)^{k}}{k !} \sum_{i=0}^{n} p_{n i}^{(m)}(x) \int_{x_{i}}^{x}\left[f^{(k+1)}(t)-P_{r}(t)\right]\left(t-x_{i}\right)^{k} d t .
$$


3844 Inequalities in polynomial interpolation

Proof. We define

$$
\begin{aligned}
v_{i}(x) & =\int_{x_{i}}^{x}\left[f^{(k+1)}(t)-P_{r}(t)\right]\left(t-x_{i}\right)^{k} d t \\
& =\int_{x_{i}}^{x} g(t)\left(t-x_{i}\right)^{k} d t,
\end{aligned}
$$

where, obviously, $g(t)=f^{(k+1)}(t)-P_{r}(t)$. We denote

$$
R_{k, r}(x)=f(x)-L_{n}(x)=\frac{(-1)^{k}}{k !} \sum_{i=0}^{n} p_{n i}(x) v_{i}(x),
$$

see Theorem 2.2. Then we have

$$
\begin{aligned}
R_{k, r}^{(m)}(x) & =\frac{(-1)^{k}}{k !} \sum_{i=0}^{n}\left[p_{n i}(x) v_{i}(x)\right]^{(m)} \\
& =\frac{(-1)^{k}}{k !} \sum_{i=0}^{n} \sum_{j=0}^{m}\left(\begin{array}{c}
m \\
j
\end{array}\right) p_{n i}^{(j)}(x) v_{i}^{(m-j)}(x) \\
& =\frac{(-1)^{k}}{k !} \sum_{i=0}^{n} p_{n i}^{(m)}(x) v_{i}(x)+\frac{(-1)^{k}}{k !} \sum_{i=0}^{n} \sum_{j=0}^{m-1}\left(\begin{array}{c}
m \\
j
\end{array}\right) p_{n i}^{(j)}(x) v_{i}^{(m-j)}(x) .
\end{aligned}
$$

We introduce the notation

$$
B(x)=\frac{(-1)^{k}}{k !} \sum_{i=0}^{n} \sum_{j=0}^{m-1}\left(\begin{array}{c}
m \\
j
\end{array}\right) p_{n i}^{(j)}(x) v_{i}^{(m-j)}(x)
$$

such that

$$
R_{k, r}^{(m)}(x)=\frac{(-1)^{k}}{k !} \sum_{i=0}^{n} p_{n i}^{(m)}(x) v_{i}(x)+B(x) .
$$

We now rewrite $B(x)$ in the form

$$
B(x)=\frac{(-1)^{k}}{k !} \sum_{i=0}^{n} \sum_{j=0}^{m-2}\left(\begin{array}{c}
m \\
j
\end{array}\right) p_{n i}^{(j)}(x) v_{i}^{(m-j)}(x)+\frac{(-1)^{k}}{k !} m \sum_{i=0}^{n} p_{n i}^{(m-1)}(x) v_{i}^{\prime}(x) .
$$

We have

$$
v_{i}^{\prime}(x)=g(x)\left(x-x_{i}\right)^{k}
$$

such that

$$
\sum_{i=0}^{n} p_{n i}^{(m-1)}(x) v_{i}^{\prime}(x)=g(x) \sum_{i=0}^{n} p_{n i}^{(m-1)}(x)\left(x-x_{i}\right)^{k}=0,
$$

for $k \geq m$-see Lemma 4.1. 
We also have

$$
v_{i}^{(m-j)}(x)=\sum_{l=0}^{m-j-1}\left(\begin{array}{c}
m-j-1 \\
l
\end{array}\right) g^{(l)}(x) \frac{k !}{(k-m+j+l+1) !}\left(x-x_{i}\right)^{k-m+j+l+1},
$$

for $m \geq j+2$ such that

$$
\begin{aligned}
\sum_{i=0}^{n} \sum_{j=0}^{m-2}\left(\begin{array}{c}
m \\
j
\end{array}\right) p_{n i}^{(j)}(x) v_{i}^{(m-j)}(x)= & \sum_{j=0}^{m-2}\left(\begin{array}{c}
m \\
j
\end{array}\right) \sum_{l=0}^{m-j-1}\left(\begin{array}{c}
m-j-1 \\
l
\end{array}\right) \frac{k !}{(k-m+j+l+1) !} \\
& \times \sum_{i=0}^{n} p_{n i}^{(j)}(x)\left(x-x_{i}\right)^{k-m+j+l+1} \\
= & 0,
\end{aligned}
$$

if $k-m+j+l+1 \geq j+1$, that is, $k \geq m$, since $l \geq 0$ - see also Lemma 4.1. Hence, $B(x)=$ 0 in all cases. Now from (4.21) it follows that

$$
\begin{aligned}
R_{k, r}^{(m)}(x) & =\frac{(-1)^{k}}{k !} \sum_{i=0}^{n} p_{n i}^{(m)}(x) v_{i}(x) \\
& =\frac{(-1)^{k}}{k !} \sum_{i=0}^{n} p_{n i}^{(m)}(x) \int_{x_{i}}^{x}\left[f^{(k+1)}(t)-P_{r}(t)\right]\left(t-x_{i}\right)^{k} d t .
\end{aligned}
$$

On the other hand, we have

$$
\left[f(x)-L_{n}(x)\right]^{(m)}=f^{(m)}(x)-L_{n}^{(m)}(x) .
$$

This completes the proof.

TheOREM 4.3. Under the assumptions of Theorem 4.2,

$$
\left|f^{(m)}(x)-L_{n}^{(m)}(x)\right| \leq \frac{E_{r}\left(f^{(k+1)}\right)}{(k+1) !} \sum_{i=0}^{n}\left|p_{n i}^{(m)}(x)\right|\left|x-x_{i}\right|^{k+1},
$$

where $E_{r}(\cdot)$ is defined by (3.6).

Proof. Let $P_{r}(t)=P_{r}^{*}(t)$, where $P_{r}^{*}(t)$ is defined by (3.6) for the function $g(t)=f^{(k+1)}(t)$. We have

$$
\begin{aligned}
\left|R_{k, r}^{(m)}(x)\right| & =\left|\frac{(-1)^{k}}{k !} \sum_{i=0}^{n} p_{n i}^{(m)}(x) \int_{x_{i}}^{x}\left[f^{(k+1)}(t)-P_{r}^{*}(t)\right]\left(t-x_{i}\right)^{k} d t\right| \\
& \leq \frac{\left\|f^{(k+1)}(t)-P_{r}^{*}(t)\right\|_{\infty}}{(k+1) !} \sum_{i=0}^{n}\left|p_{n i}^{(m)}(x)\right|\left|x-x_{i}\right|^{k+1} \\
& =\frac{E_{r}\left(f^{(k+1)}\right)}{(k+1) !} \sum_{i=0}^{n}\left|p_{n i}^{(m)}(x)\right|\left|x-x_{i}\right|^{k+1},
\end{aligned}
$$


since

$$
\left|\int_{x_{i}}^{x}\left(t-x_{i}\right)^{k} d t\right|=\frac{\left|x-x_{i}\right|^{k+1}}{k+1} .
$$

TheOREM 4.4. Under the assumptions of Theorem 3.3 and Lemma 4.1,

$$
\begin{aligned}
& \left|f^{(m)}(x)-L_{n}^{(m)}(x)\right| \leq \frac{\Gamma_{k+1}-\gamma_{k+1}}{2(k+1) !} \sum_{i=0}^{n}\left|p_{n i}^{(m)}(x)\right|\left|x-x_{i}\right|^{k+1}, \\
& \left|f^{(m)}(x)-L_{n}^{(m)}(x)\right| \leq \frac{1}{k !} \sum_{i=0}^{n}\left(S_{k i}-\gamma_{k+1}\right)\left|p_{n i}^{(m)}(x)\right|\left|x-x_{i}\right|^{k+1}, \\
& \left|f^{(m)}(x)-L_{n}^{(m)}(x)\right| \leq \frac{1}{k !} \sum_{i=0}^{n}\left(\Gamma_{k+1}-S_{k i}\right)\left|p_{n i}^{(m)}(x)\right|\left|x-x_{i}\right|^{k+1} .
\end{aligned}
$$

Proof. We choose $P_{r}(t)=\Gamma_{k+1}+\gamma_{k+1} / 2$ in Theorem 4.2. Then we get

$$
\begin{aligned}
\left|f^{(m)}(x)-L_{n}^{(m)}(x)\right| & \leq \frac{1}{k !} \sum_{i=0}^{n}\left|p_{n i}^{(m)}(x)\right|\left|\int_{x_{i}}^{x}\left[f^{(k+1)}(t)-\frac{\Gamma_{k+1}+\gamma_{k+1}}{2}\right]\left(t-x_{i}\right)^{k} d t\right| \\
& \leq \frac{\Gamma_{k+1}-\gamma_{k+1}}{2(k !)} \sum_{i=0}^{n}\left|p_{n i}^{(m)}(x)\right|\left|\int_{x_{i}}^{x}\left(t-x_{i}\right)^{k} d t\right| \\
& =\frac{\Gamma_{k+1}-\gamma_{k+1}}{2(k+1) !} \sum_{i=0}^{n}\left|p_{n i}^{(m)}(x)\right|\left|x-x_{i}\right|^{k+1} .
\end{aligned}
$$

If we choose $P_{r}(t)=\gamma_{k+1}$ in Theorem 4.2, then we get

$$
\begin{aligned}
\left|f^{(m)}(x)-L_{n}^{(m)}(x)\right| & \leq \frac{1}{k !} \sum_{i=0}^{n}\left|p_{n i}^{(m)}(x)\right|\left|\int_{x_{i}}^{x}\left[f^{(k+1)}(t)-\gamma_{k+1}\right]\left(t-x_{i}\right)^{k} d t\right| \\
& \leq \frac{1}{k !} \sum_{i=0}^{n}\left(S_{k i}-\gamma_{k+1}\right)\left|p_{n i}^{(m)}(x)\right|\left|x-x_{i}\right|^{k+1},
\end{aligned}
$$

since $\left|\int_{x_{i}}^{x}\left[f^{(k+1)}(t)-\gamma_{k+1}\right] d t\right|=\left|f^{(k)}(x)-f^{(k)}\left(x_{i}\right)-\gamma_{k+1}\left(x-x_{i}\right)\right|$.

In a similar way we prove that the third inequality holds.

\section{References}

[1] R. P. Agarwal and P. J. Y. Wong, Error Inequalities in Polynomial Interpolation and Their Applications, Mathematics and Its Applications, vol. 262, Kluwer Academic, Dordrecht, 1993.

[2] P. Cerone and S. S. Dragomir, Midpoint-type rules from an inequalities point of view, Handbook of Analytic-Computational Methods in Applied Mathematics (G. Anastassiou, ed.), Chapman \& Hall/CRC, Florida, 2000, pp. 135-200.

[3] _ Trapezoidal-type rules from an inequalities point of view, Handbook of AnalyticComputational Methods in Applied Mathematics (G. Anastassiou, ed.), Chapman \& Hall/CRC, Florida, 2000, pp. 65-134.

[4] X.-L. Cheng, Improvement of some Ostrowski-Grüss type inequalities, Comput. Math. Appl. 42 (2001), no. 1-2, 109-114. 
[5] S. S. Dragomir, R. P. Agarwal, and P. Cerone, On Simpson's inequality and applications, J. Inequal. Appl. 5 (2000), no. 6, 533-579.

[6] S. S. Dragomir and S. Wang, An inequality of Ostrowski-Grüss type and its applications to the estimation of error bounds for some special means and for some numerical quadrature rules, Comput. Math. Appl. 33 (1997), no. 11, 15-20.

[7] H. N. Mhaskar and D. V. Pai, Fundamentals of Approximation Theory, CRC Press, Florida; Narosa, New Delhi, 2000.

[8] D. S. Mitrinović, J. E. Pečarić, and A. M. Fink, Classical and New Inequalities in Analysis, Mathematics and Its Applications (East European Series), vol. 61, Kluwer Academic, Dordrecht, 1993.

[9] C. E. M. Pearce, J. E. Pečarić, N. Ujević, and S. Varošanec, Generalizations of some inequalities of Ostrowski-Grüss type, Math. Inequal. Appl. 3 (2000), no. 1, 25-34.

[10] N. Ujević, Error inequalities for a corrected interpolating polynomial, New York J. Math. 10 (2004), 69-81.

[11] Error inequalities for a perturbed interpolating polynomial, Nonlinear Stud. 12 (2005), no. 3, 233-245.

[12] N. Ujević and A. J. Roberts, A corrected quadrature formula and applications, ANZIAM J. 45 (2004), no. (E), E41-E56.

Nenad Ujević: Department of Mathematics, University of Split, Teslina 12/III, 21000 Split, Croatia E-mail address: ujevic@pmfst.hr 


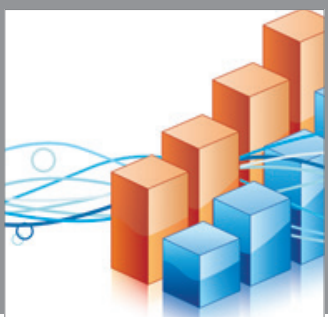

Advances in

Operations Research

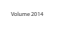

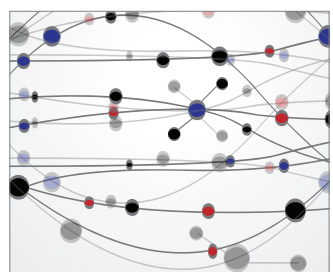

\section{The Scientific} World Journal
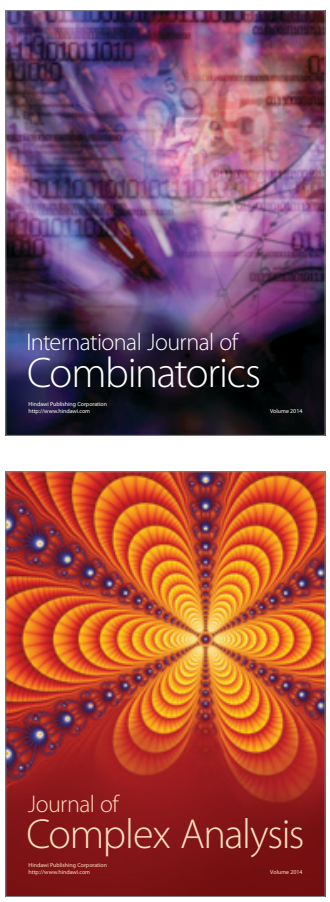

International Journal of

Mathematics and

Mathematical

Sciences
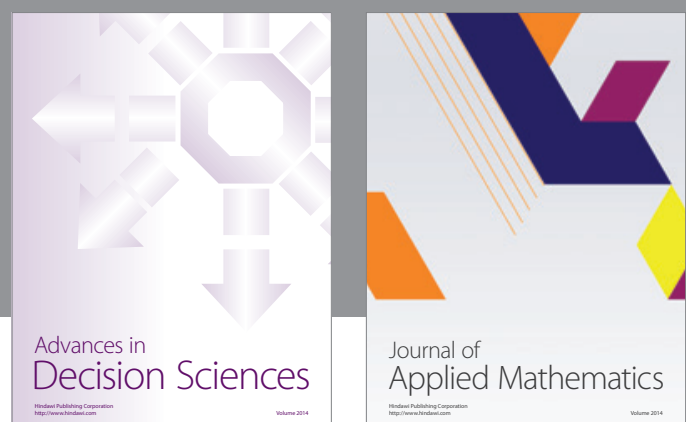

Journal of

Applied Mathematics
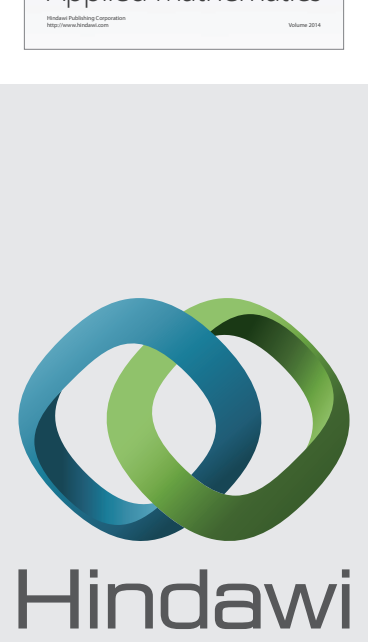

Submit your manuscripts at http://www.hindawi.com
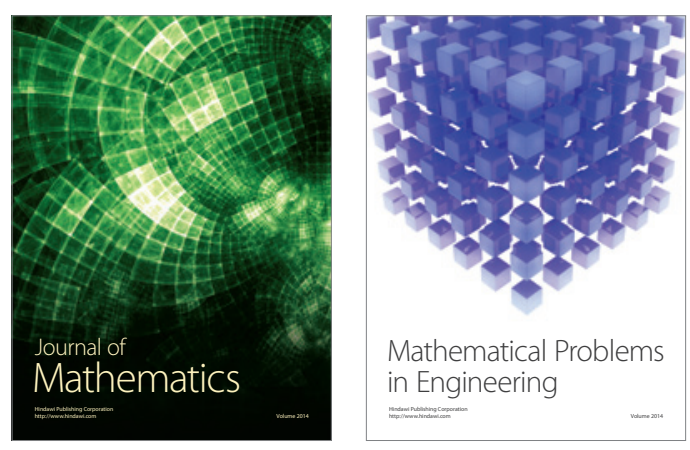

Mathematical Problems in Engineering
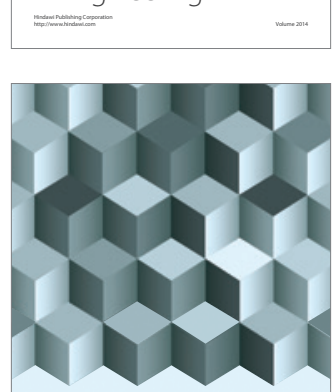

Journal of

Function Spaces
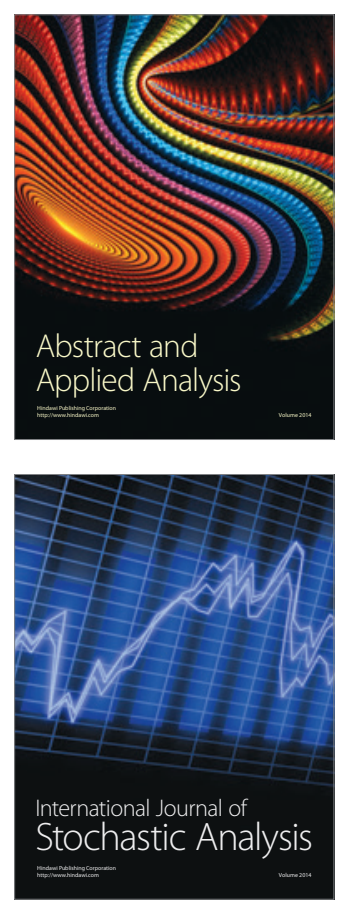

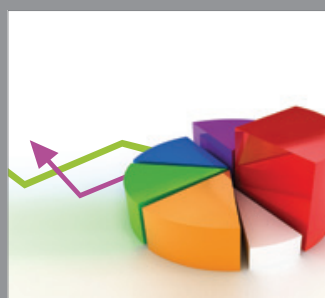

ournal of

Probability and Statistics

Promensencen
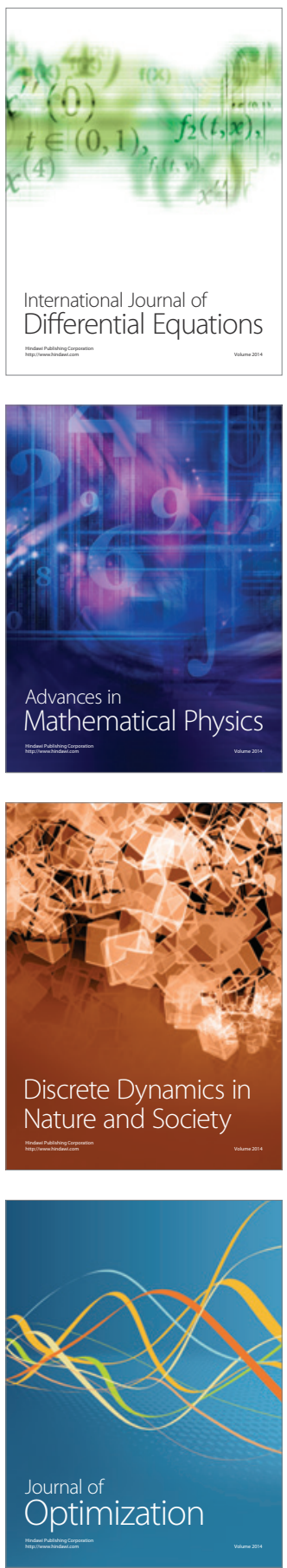